\title{
CORE: A Clustering Optimization algorithm for Resource Efficiency in LTE-A Networks
}

\author{
Georgios Kollias*, Ferran Adelantado ${ }^{\dagger}$, Kostas Ramantas*, Christos Verikoukis ${ }^{\ddagger}$ \\ *Iquadrat Informatica, Barcelona, Spain \\ ${ }^{\dagger}$ Universitat Oberta de Catalunya (UOC), Barcelona, Spain \\ ${ }^{\ddagger}$ Centre Tecnologic de Telecomunicacions de Catalunya (CTTC), Barcelona, Spain \\ Email: gkollias@iquadrat.com, ferranadelantado@uoc.edu, kramantas@iquadrat.com,cveri@cttc.es
}

\begin{abstract}
In a fluctuating mobile environment where operators have to confront the ever increasing demands of their subscribers, insufficient spectrum poses capacity limitations. This is more evident in the downlink (DL) direction, since DL resources are over-utilized compared to the uplink (UL) ones as a result of asymmetry in the generated traffic and intense interference. In this framework, we propose the creation of Device-to-Device (D2D) based clusters of users where intra-cluster communication will be achieved over UL resources. The minimization of the required resources (equivalent to the maximization of the spectral efficiency), is formulated as an integer (binary) linear optimization problem. Finally, a low-complexity clustering optimization algorithm for resource efficiency (CORE), is devised. Illustrative results prove that CORE, manages to increase the spectral efficiency and the network's capacity.
\end{abstract}

Index Terms-Device-to-Device communication, LTE-A, asymmetry traffic, clustering,

\section{INTRODUCTION}

The booming demands of cellular users [1] due to introduction of smart devices, along with the ever increasing number of mobile subscriptions, bring operators' capacity to their limits. As it has been seen [2], densifying networks with small cells is a solution adopted recently by mobile operators to cope with increased demands. In this framework, and in light of the envisaged increased trend on number of users, a debate on whether this amount of users could be considered as part of Radio Access Network (RAN), complementary to the fixed nodes provided by operators, has initiated [3].

In that sense, the usage of short-range communications (i.e Device-to-Device (D2D)) among adjacently located user equipments (UEs), labelled as proximity services by 3GPP [4], could be a decisive step towards facing the need for higher capacity and improved data rates. However, the impact of proximity services is limited and the utilization of short-range communications among D2D UEs should not be restricted to them. Recent works show that the introduction of D2D communications in multicast groups of UEs [5]-[7] enhances the base station's multicast rate, usually determined by the worst channel quality inside the group. For instance, the authors in [5], manage to derive closed-form expressions for selecting the optimal number of D2D retransmitters in a multicast group. Similarly, Condoluci et al. [6] exploit D2D communication in multicast services groups. Their proposed D2D-supported Conventional Multicast Scheme improves the transmission rate of the multicast groups. This is achieved by serving UEs with poor connection to the eNodeB (eNB), through D2D links. In other case, these UEs would limit the data rate at which each group of UEs is served, since the rate is defined by the UE with the worst channel condition. Finally, aligned with the previous, the authors in [7], analyze the key aspects of network controlled D2D-based multicasting (e.g.TDD and FDD combination for multicast/unicast nodes or cooperative transmission within multicast groups). The proposal enhances the throughput and reduces the delay of the multicast group. Unlike the previous works that define the cluster as a group of UEs that receive a multicast service, the work in [8] uses the term clustering to refer to D2D pairs that re-utilize the same resources. It proposes a joint lowcomplexity D2D power control and clustering mechanism that minimizes the co-channel interference, thereby reducing the outage probability of the D2D pairs.

It is worth pointing out that in the aforementioned works D2D operations are controlled by the network. Specifically, it is assumed that the overlaid eNB selects the cluster heads, manages the interference, performs the power control, and schedules D2D transmissions. Motivated by the above, this work makes one step further and combines the concepts of short-range communications and clustering to a greater extend. As a result, we propose clustering of UEs under the coordination of a UE, hereafter denoted as Cluster-Head $(\mathrm{CH})$, which is characterized by a good connection with the overlaid eNB. Clusters will be created and managed in a hybrid distributed-centralized manner. The main objective of the proposed clustering algorithm is the reduction of the resources' utilization, and hence the increase of the achievable capacity. Specifically, an initial NP-hard optimization problem is defined to subsequently propose a low complexity algorithm.

The rest of the paper is organized as follows: The problem under study is presented in Section II while Section III describes the system model. Our optimization problem for minimizing utilization of resources is studied in Section IV. Finally, numerical results are presented in Section $\mathrm{V}$ while Section VI concludes the paper.

\section{Problem Formulation}

A UE inside the coverage area of an eNB may experience bad signal quality as a result of factors such as multi-path fading, interference and non-line of sight. In these situations, two things might happen; the eNB will have to transmit at 
lower Modulation and Coding Scheme (MCS) to serve UE's demands, thus consuming more resources, or the UE will get no service. In order to improve the service delivered to such UEs, we propose the creation of D2D-based clusters. The clusters, composed of two or more UEs, are created on the basis of good quality links between the $\mathrm{CH}$ and the eNB, and between the $\mathrm{CH}$ and the rest of the Cluster Members (CM) (see Fig. 1).

Although the system is FDD, D2D communications within the cluster (from the $\mathrm{CH}$ to the $\mathrm{CMs}$ and vice versa) are conducted over the UL resources [9], allocated in an orthogonal manner with resources allocated in the UL for cellular UEs, to reduce the interference incurred in neighboring cells and to exploit the imbalance between the UL and the DL loads [10]. In turn, the power transmitted within the cluster is also lower to mitigate the potential interference. As for the operation of the clusters, CMs transmit their UL traffic to the $\mathrm{CH}$, which subsequently forwards it to the eNB. In the opposite link, the $\mathrm{CH}$ receives the DL traffic of all the CMs from the eNB, and then forwards it to the corresponding CM.

The seamless operation of the cluster is achieved with the network assistance. Specifically, resources required for the communication of the $\mathrm{CMs}$ and the $\mathrm{CH}$ within the cluster are scheduled by the eNB. Thus, the eNB allocates UL resources to each cluster. Within the cluster, the $\mathrm{CH}$ is responsible for scheduling the resources among the CMs. However, the implementation of the scheduling algorithms falls beyond the scope of this work.

It is worth noting that the expected spectral efficiency improvement is twofold. On the one hand, low quality links (with higher resources requirements) are substituted by two higher quality links. On the other hand, the high DL load (which is usually the limiting link) is reduced at the expense of increasing the UL load (usually less loaded). Such transfer of traffic from the DL to the UL reduces the DL/UL imbalance, thereby increasing the achievable capacity.

The success in improving the spectral efficiency does, however, depend on the configuration of the clusters. Specifically, the key aspects are: i) when to create a cluster, ii) how many UEs should take part of it, and iii) which UE should play the role of $\mathrm{CH}$. All these issues are analyzed in the sequel.

\section{System Model}

The scenario is composed of a set of UEs, namely $\mathcal{U}$, distributed over an area covered by a set $\mathcal{B}$ of eNBs. As in LTE the cell selection procedure associates each UE with the eNB from which it receives the highest RSRP (Reference Signal Received Power) or RSRQ (Reference Signal Received Quality), we define $\mathcal{U}_{k}$ as the subset of UEs $i \in \mathcal{U}$ associated to eNB $k \in \mathcal{B}$, where $\bigcap_{k} \mathcal{U}_{k}=\emptyset$ and $\bigcup_{k} \mathcal{U}_{k}=\mathcal{U}$.

The traffic of a UE $i$ is henceforth characterized by its DL required transmission rate, $R_{i}^{D L}$, and its UL required transmission rate, referred to as $R_{i}^{U L}=\alpha_{i} R_{i}^{D L}$, with $\alpha_{i} \geq 0$. In general, DL traffic is more intense than UL traffic. Therefore, despite not being an actual constraint, $\alpha_{i}$ usually takes values between 0 and 1 . In the following full-duplex radios are assumed [11]. Frequent notation used in the sequel is summarized in Table I.

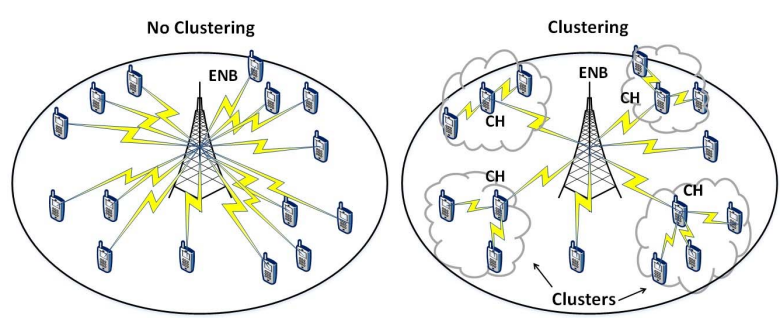

Fig. 1: Scenario without and with clustering

TABLE I: Frequent notation

\begin{tabular}{cl}
\hline Notation & Description \\
\hline $\mathcal{U}$ & Set of UEs \\
$\mathcal{B}$ & Set of eNBs \\
$\mathcal{U}_{k}$ & Set of UEs connected to the eNB $k$ \\
$\mathcal{C}_{u}$ & Set of UEs that belong to cluster $u$ \\
$h_{u}$ & CH of the cluster $u$ \\
$\mathcal{C}^{k}$ & Set of UEs in clusters associated to eNB $k$ \\
$\mathcal{C}^{k}$ & Set of UEs outside clusters and associated to eNB $k$ \\
$\mathcal{C}^{k *}$ & Set of UEs directly connected to eNB $k$ (including \\
$R_{i}^{D L}, R_{i}^{U L}$ & CHs) \\
$\alpha_{i}$ & Required transmission rate for UE $i$ in DL and UL \\
$T_{s}$ & $\frac{R_{i}^{U L}}{R_{i}^{D L}}$ \\
$\Gamma_{i}^{k}$ & Subframe time \\
$\gamma_{i}^{k}$ & SINR in the DL from eNB $k$ to UE $i$ \\
$\gamma_{j i}$ & SINR in the UL from UE $i$ to eNB $k$ \\
$N_{k}^{D L}{ }^{\prime}, N_{k}^{U L} L^{\prime}$ & SINR from UE $j$ to UE $i$ \\
$N_{k}^{D L}, N_{k}^{U L}$ & RBs required by eNB $k$ in DL/UL without clustering \\
$S_{i}^{k}(\cdot), S_{j, i}(\cdot)$ & RBs required by eNB $k$ in DL/UL with clustering \\
& Transport Block Size in transmission from/to $i \in \mathcal{U}_{k}$ \\
$\Phi_{i}^{k}, \phi_{i}^{k}, \phi_{j i}$ & and $j$, respectively \\
$G_{i j}^{k}=\frac{T_{s}}{S_{i}^{k}\left(\Gamma_{i}^{k}\right)}, \phi_{i}^{k}=\frac{T_{s}}{S_{i}^{k}\left(\gamma_{i}^{k}\right)}, \phi_{j i}=\frac{T_{s}}{S_{j i}\left(\gamma_{j i}\right)}$ \\
$\mathcal{H}_{i}$ & Gain achieved by UE $i$ when clustering with UE $j$ \\
& Set of UEs with which $i$ could create a cluster \\
\hline
\end{tabular}

\section{A. Resources required without clustering}

In LTE, the MCS used in a transmission is selected according to the signal to interference plus noise ratio (SINR). Accordingly, the number of bits transmitted by UE $i$ during a subframe time $\left(T_{s}=1 \mathrm{~ms}\right)$, defined as Transport Block Size (TBS) [12], depends on the SINR as well. Let $\Gamma_{i}^{k}$ denote the SINR in the DL of a UE $i$ associated with eNB $k$ and $S_{i}^{k}\left(\Gamma_{i}^{k}\right)$ the corresponding TBS. According to [13] the transmission rate of a particular MCS can be approximated by an attenuated and truncated form of the Shannon bound, and thus

$$
S_{i}^{k}\left(\Gamma_{i}^{k}\right)=T_{s} r W \log _{2}\left(1+\Gamma_{i}^{k}\right)
$$

where $r$ is the attenuating factor and $W$ is the bandwidth of a Physical Resource Block (PRB). Likewise, if the SINR in the $\mathrm{UL}$ is denoted by $\gamma_{i}^{k}$, the corresponding TBS is then

$$
S_{i}^{k}\left(\gamma_{i}^{k}\right)=T_{s} r W \log _{2}\left(1+\gamma_{i}^{k}\right)
$$

Based on the definitions stated above, the average number of RBs per subframe required in the DL and in the UL of eNB $k$ are given by

$$
N_{k}^{D L^{\prime}}=\sum_{i \in \mathcal{U}_{k}} \frac{R_{i}^{D L} T_{s}}{S_{i}^{k}\left(\Gamma_{i}^{k}\right)}=\sum_{i \in \mathcal{U}_{k}} R_{i}^{D L} \Phi_{i}^{k}
$$


and

$$
N_{k}^{U L^{\prime}}=\sum_{i \in \mathcal{U}_{k}} \frac{R_{i}^{U L} T_{s}}{S_{i}^{k}\left(\gamma_{i}^{k}\right)}=\sum_{i \in \mathcal{U}_{k}} \alpha_{i} R_{i}^{D L} \phi_{i}^{k}
$$

where, for simplicity, $\Phi_{i}^{k}=\frac{T_{s}}{S_{i}^{k}\left(\Gamma_{i}^{k}\right)}$ and $\phi_{i}^{k}=\frac{T_{s}}{S_{i}^{k}\left(\gamma_{i}^{k}\right)}$.

\section{B. Resources required with clustering}

Before proceeding with the analysis, and for a given cluster $u$, we define the set of UEs which are members of the cluster as $\mathcal{C}_{u}$, and the $\mathrm{CH}$ as $h_{u} \in \mathcal{C}_{u}$. The eNB to which a cluster $u$ is connected is determined by the cluster head, $h_{u}$. Thus, we define the set of clusters connected to eNB $k$ as $\mathcal{C}^{k}=\cup_{u} \mathcal{C}_{u}$ subject to $h_{u} \in \mathcal{U}_{k}$. It is worth noting that the complement of $\mathcal{C}^{k}$ (i.e. $\overline{\mathcal{C}^{k}}=\mathcal{U}_{k} \backslash \mathcal{C}^{k}$ ) stands for the set of UEs outside any cluster that are associated to $k$. Finally, the set of UEs directly connected to the eNB $k$ (both $\mathrm{CHs}$ and UEs outside the clusters) is referred to as $\mathcal{C}^{k *}=\overline{\mathcal{C}^{k}} \cup\left\{h_{u}\right\}_{\forall h_{u} \in \mathcal{U}_{k}}$.

When the clustering of UEs takes place, the usage of DL and UL resources differs substantially. Specifically, DL is only used in communications from eNBs to UEs, whereas UL resources' usage is divided in: i) UL transmissions from UEs to the eNBs, and ii) transmissions within the cluster. In the following the analysis of the resources required in each link after the creation of clusters is presented.

1) DL resources: For a given eNB $k$, DL resources are used to communicate $k$ with all the UEs in $\mathcal{C}^{k *}$. Thus, whereas UEs outside the clusters (i.e. UEs in $\overline{\mathcal{C}^{k}}$ ) require the same amount of resources as in Section III-A, CHs are responsible for receiving the DL traffic of all the UEs within their cluster. This concept can be easily formulated as

$$
N_{k}^{D L}=\sum_{i \in \overline{\mathcal{C}^{k}}} R_{i}^{D L} \Phi_{i}^{k}+\sum_{\mathcal{C}_{u} \in \mathcal{C}^{k}} \sum_{i \in \mathcal{C}_{u}} R_{i}^{D L} \Phi_{h_{u}}^{k}
$$

The first term in (5) stands for the number of DL resources required by the non-cluster UEs and the second term is the number of resources required by the $\mathrm{CMs}$ (including the $\mathrm{CHs}$ ).

2) UL resources: Resources required in the UL can be split up into resources used outside the clusters $\left(N_{\mathcal{C}^{k *}}^{U L}\right)$ and resources used within the clusters $\left(N_{\mathcal{C}_{u}}^{U L}\right)$.

$$
N_{k}^{U L}=N_{\mathcal{C}^{k *}}^{U L}+\sum_{\mathcal{C}_{u} \in \mathcal{C}^{k}} N_{\mathcal{C}_{u}}^{U L}
$$

Analogously to (5), the resources required outside the cluster, $N_{\mathcal{C}^{k *}}^{U L}$, can be written as,

$$
N_{\mathcal{C}^{k *}}^{U L}=\sum_{i \in \overline{\mathcal{C}^{k}}} \alpha_{i} R_{i}^{D L} \phi_{i}^{k}+\sum_{\mathcal{C}_{u} \in \mathcal{C}^{k}} \sum_{i \in \mathcal{C}_{u}} \alpha_{i} R_{i}^{D L} \phi_{h_{u}}^{k}
$$

With regard to the resources required within a cluster $u$, $N_{\mathcal{C}_{u}}^{U L}$, they in turn may be expressed as the sum of resources used in transmissions from the $\mathrm{CH}$ (i.e. $h_{u}$ ) to the rest of the $\mathrm{CMs}, N_{\mathcal{C}_{u}}^{C H}$, and resources used in transmissions from all the CMs to the $\mathrm{CH}, N_{\mathcal{C}_{u}}^{C M}$.

$$
N_{\mathcal{C}_{u}}^{U L}=N_{\mathcal{C}_{u}}^{C H}+N_{\mathcal{C}_{u}}^{C M}
$$

Note that, within the cluster, CMs will transmit their UL traffic to the $\mathrm{CH}$, whereas the $\mathrm{CH}$ will forward the $\mathrm{CMs}$ ' DL traffic received from the eNB. Accordingly, the resources used to transmit from the $\mathrm{CH}$ to the rest of the $\mathrm{CMs}$ can be expressed as

$$
N_{\mathcal{C}_{u}}^{C H}=\sum_{i \in \mathcal{C}_{u} \backslash\left\{h_{u}\right\}} \frac{R_{i}^{D L} T_{s}}{S_{h_{u} i}\left(\gamma_{h_{u} i}\right)}=\sum_{i \in \mathcal{C}_{u} \backslash\left\{h_{u}\right\}} R_{i}^{D L} \phi_{h_{u} i}
$$

where $\gamma_{h_{u} i}$ is the SINR received by UE $i$ upon a transmission from $h_{u}$, and $S_{h_{u} i}\left(\gamma_{h_{u} i}\right)=T_{s} r W \log _{2}\left(1+\gamma_{h_{u} i}\right)$. As for $N_{\mathcal{C}_{u}}^{C M}$

$$
N_{\mathcal{C}_{u}}^{C M}=\sum_{i \in \mathcal{C}_{u} \backslash\left\{h_{u}\right\}} \frac{\alpha_{i} R_{i}^{D L} T_{s}}{S_{i h_{u}}\left(\gamma_{i h_{u}}\right)}=\sum_{i \in \mathcal{C}_{u} \backslash\left\{h_{u}\right\}} \alpha_{i} R_{i}^{D L} \phi_{i h_{u}}
$$

where $\gamma_{i h_{u}}$ is the SINR received by the $\mathrm{CH} h_{u}$ upon a transmission from UE $i$, and $S_{i h_{u}}\left(\gamma_{i h_{u}}\right)=T_{s} r W \log _{2}\left(1+\gamma_{i h_{u}}\right)$. Hence, by using (7), (8), (9) and (10) in (6), the total average number of resources per subframe required in the UL with clustering is given by

$$
\begin{aligned}
N_{k}^{U L} & =\sum_{i \in \overline{\mathcal{C}^{k}}} \alpha_{i} R_{i}^{D L} \phi_{i}^{k}+\sum_{\mathcal{C}_{u} \in \mathcal{C}^{k}}\left[\alpha_{h_{u}} R_{h_{u}}^{D L} \phi_{h_{u}}^{k}+\right. \\
& \left.+\sum_{i \in \mathcal{C}_{u} \backslash h_{u}} R_{i}^{D L}\left(\alpha_{i} \phi_{h_{u}}^{k}+\phi_{h_{u} i}+\alpha_{i} \phi_{i h_{u}}\right)\right]
\end{aligned}
$$

\section{Clustering Optimization}

\section{A. Optimal Solution}

The optimal clustering configuration is such that it achieves the highest spectral efficiency, or equivalently the minimum utilization of resources. According to (5) and (6), the total average number of RBs per subframe required with clustering in eNB $k$ may be calculated as

$$
\begin{aligned}
N_{k}^{T} & =N_{k}^{D L}+N_{k}^{U L} \\
& =\sum_{i \in \mathcal{C}^{k *}} R_{i}^{D L}\left(\Phi_{i}^{k}+\alpha_{i} \phi_{i}^{k}\right)+ \\
& +\sum_{\mathcal{C}_{u} \in \mathcal{C}^{k}} \sum_{i \in \mathcal{C}_{u} \backslash\left\{h_{u}\right\}} R_{i}^{D L}\left(\phi_{h_{u} i}+\alpha_{i} \phi_{i h_{u}}+\alpha_{i} \phi_{h_{u}}^{k}+\Phi_{h_{u}}^{k}\right)
\end{aligned}
$$

and therefore, the total number of resources used in the scenario under study is given by $N^{T}=\sum_{k \in \mathcal{B}} N_{k}^{T}$.

The eNB plays an important role in the coordination of the clusters' creation, as it will be explained in Section IV-C. Therefore, let us assume that, for a given cluster $u$ with $h_{u} \in$ $\mathcal{U}_{k}$, all UEs are associated with eNB $k$ in case the cluster is not created, i.e. $\forall i \in \mathcal{C}_{u}$ where $h_{u} \in \mathcal{U}_{k}$, then $i \in \mathcal{U}_{k}$. In other words, the creation of clusters is restricted to UEs associated with the same eNB. Thus, the optimal clustering configuration is achieved by minimizing the number of required RBs in each eNB, i.e. $N_{k}^{T}$.

We define the eNB association vector $\mathbf{x}^{k}=$ $\left(x_{1}^{k}, \ldots, x_{n}^{k}\right)^{T} \in\{0,1\}^{n}$, where $n$ is the number of UEs in the coverage area of eNB $k\left(n=\left|\mathcal{U}_{k}\right|\right.$, where $|\cdot|$ stands for the cardinality operator). Specifically, $x_{i}^{k}=1$ if UE $i \in \mathcal{U}_{k}$ is directly connected to eNB $k\left(i \in \mathcal{C}^{k *}\right)$. Conversely, $x_{i}^{k}=0$ if $i \notin \mathcal{C}^{k *}$. In parallel, we define the D2D association matrix $\mathbf{y}^{k} \in\{0,1\}^{n \times n}$, where $y_{i j}^{k}=1$ if UE $j$ is the $\mathrm{CH}$ of a cluster that contains UE $i$. Otherwise, $y_{i j}^{k}=0$. According to 
(12), the total number of RBs required in $k$ may be re-written as

$$
\begin{aligned}
N_{k}^{T}\left(\mathbf{x}^{k}, \mathbf{y}^{k}\right) & =\sum_{i \in \mathcal{U}_{k}} x_{i}^{k} R_{i}^{D L}\left(\Phi_{i}^{k}+\alpha_{i} \phi_{i}^{k}\right)+ \\
& +\sum_{i \in \mathcal{U}_{k}} \sum_{j \in \mathcal{U}_{k} \backslash\{i\}} y_{i j}^{k} R_{i}^{D L}\left(\phi_{h_{u} i}+\alpha_{i} \phi_{i h_{u}}+\right. \\
& \left.+\alpha_{i} \phi_{h_{u}}^{k}+\Phi_{h_{u}}^{k}\right)
\end{aligned}
$$

The optimal association vectors $\mathbf{x}^{k}$ and $\mathbf{y}^{k}$ are obtained by solving the following problem:

$$
\begin{array}{ll}
\underset{\mathbf{x}^{k}, \mathbf{y}^{k}}{\operatorname{minimize}} & N_{k}^{T}\left(\mathbf{x}^{k}, \mathbf{y}^{k}\right) \\
\text { s.t. } & x_{i}^{k} \in\{0,1\}, \forall i \in \mathcal{U}_{k} \\
& y_{j i}^{k} \in\{0,1\}, \forall i, j \in \mathcal{U}_{k} \\
& \sum_{i \in \mathcal{U}_{k}} x_{i}^{k} \geq 1 \\
& \sum_{j \in \mathcal{U}_{k}} y_{j i}^{k} \leq 1, \forall i \in \mathcal{U}_{k} \\
& x_{i}^{k}+\sum_{j \in \mathcal{U}_{k} \backslash\{i\}} y_{i j}^{k}=1, \forall i \in \mathcal{U}_{k} \\
& y_{i i}^{k}=0, \forall i \in \mathcal{U}_{k}
\end{array}
$$

The optimization problem is an integer (binary) linear program (ILP) (14a)-(14b) where at least one of the UEs must be directly connected to an overlaid eNB (14c), and none of the UEs can be member of more than one cluster (14d). Finally, all UEs must be connected either to the eNB or to a $\mathrm{CH}(14 \mathrm{e})$.

\section{B. Near Optimal Solution}

The optimization problem stated in (14) is NP-hard. In order to overcome such complexity, in this section a low complexity algorithm, $O\left(n^{3}\right)$, namely Clustering Optimization for Resource Efficiency (CORE), is presented.

Lemma 1. The creation of a cluster with two UEs, $i, j \in \mathcal{U}_{k}$, will reduce the usage of resources if $\left(\Phi_{i}^{k}-\Phi_{j}^{k}-\phi_{j i}\right)-\alpha_{i}\left(\phi_{j}^{k}+\right.$ $\left.\phi_{i j}-\phi_{i}^{k}\right)>0$, and as long as $U E j$ is the $\mathrm{CH}$.

Proof. Let us define the gain in RBs achieved in eNB $k$ after the clustering process as

$$
\begin{aligned}
G^{k} & =\left(N_{k}^{D L^{\prime}}+N_{k}^{U L^{\prime}}\right)-\left(N_{k}^{D L}+N_{k}^{U L}\right) \\
& =\sum_{\mathcal{C}_{u} \in \mathcal{C}^{k}} \sum_{i \in C_{u} \backslash\left\{h_{u}\right\}} G_{i h_{u}}^{k} \\
& =\sum_{\mathcal{C}_{u} \in \mathcal{C}^{k}} \sum_{i \in C_{u} \backslash\left\{h_{u}\right\}} R_{i}^{D L}\left[\left(\Phi_{i}^{k}-\Phi_{h_{u}}^{k}-\phi_{h_{u} i}\right)\right. \\
& \left.-\alpha_{i}\left(\phi_{h_{u}}^{k}+\phi_{i h_{u}}-\phi_{i}^{k}\right)\right]
\end{aligned}
$$

The total gain is then given by $G=\sum_{k \in \mathcal{B}} G^{k}$. As the total gain is the aggregation of the gains achieved by each UE $i$ clustered with a given $\mathrm{CH} h_{u}, G_{i h_{u}}^{k}$, the maximum gain (i.e. the minimum usage of resources) is only achieved when all the individual gains are positive. Therefore, when $G_{i h_{u}}^{k}>0$.

Lemma 2. The set of possible CHs of a $U E i \in \mathcal{U}_{k}$, namely $\mathcal{H}_{i}$, is defined as the set of UEs $j \in \mathcal{U}_{k}$ with which $G_{i j}^{k}>0$.
If, for a pair of UEs $i, j \in \mathcal{U}_{k}, G_{i j}^{k}>0$ and $\mathcal{H}_{j}=\emptyset$, then UEs $i$ and $j$ will create a cluster where $j$ will be the $C H$. Conversely, if $\mathcal{H}_{j} \neq \emptyset$, UEs $i$ and $j$ will create a cluster if $G_{i j}^{k}>G_{j n}^{k}+G_{i t}^{k}$, for $\forall n \in \mathcal{H}_{j}$ and $\forall t \in \mathcal{H}_{i}$.

Proof. As stated in Lemma 1, the gain achieved by clustering equals the aggregation of the individual gains. Therefore, Lemma 2 can be directly derived from (15).

CORE (see Algorithm 1) is based on lemmas 1 and 2. In particular, the algorithm is initialized by calculating $G_{i j}^{k}$ for UEs in the coverage area of eNB $k$ and creating the corresponding sets of possible $\mathrm{CHs}$ (line 1).

There are some UEs that get no additional gain in becoming CMs (i.e. $\mathcal{H}_{i}=\emptyset$ ), so they stay connected to eNB. In a first step of the algorithm, clusters in which $\mathrm{CHs}$ have $\mathcal{H}_{i}=\emptyset$ are created (lines 3-14). Subsequently, in a second phase (lines 15-29), conflicting situations between UEs that could be either simple $\mathrm{CMs}$ or $\mathrm{CHs}$ are resolved. Specifically, the most beneficial association in terms of resources' usage is investigated for these UEs.

\section{Implementation Issues}

The implementability of CORE is based on the availability of $\Phi_{i}^{k}, \phi_{i}^{k}$ and $\phi_{i j}$. In LTE-A, all these parameters can be directly calculated from the corresponding SINR or Channel Quality Indicator (CQI). In the case of $\phi_{i}^{k}$, it is calculated by the eNB and made available to the UE through the Downlink Control Information (DCI) sent in PDCCH ${ }^{1}$. As for $\Gamma_{i}^{k}$, it is calculated by the UE, which informs the eNB about the CQI over the PUCCH or PUSCH [12].

For a UE $j$, the availability of $\phi_{i j}$ is more complex, since it should eavesdrop the uplink Sounding Reference Signal (SRS) of the UE $i$ [14]. The feasibility and efficiency in obtaining $\phi_{i j}$ relies on the eNB assistance. Since UL transmissions of UE $i$ are scheduled by the eNB, UE $j$ may be informed by the eNB about the PRBs allocated to $j$ via PDCCH. In order to make the process efficient, the eNB only informs UE $i$ about the UEs with a similar Angle of Arrival (AoA $)^{2}$ and with a good quality channel.

With the information detailed above, UEs calculate in a distributed manner a reduced set of $G_{i j}^{k}$ values (i.e. UEs with $G_{i j}^{k}>0$ ). These values are transferred to the eNB, where CORE is executed. It is worth noting that the distributed calculation of possible gains $\left(G_{i j}^{k}\right)$ reduces drastically the additional exchanged information and simplifies the execution of CORE in the eNB.

\section{Numerical Results}

In this section we evaluate the performance of our clustering proposal with both numerical, obtained via Matlab, and simulation results. The simulation area consists of a macro cell with radius $250 \mathrm{~m}$ and the first interfering ring. We consider two different scenarios in our study: the Uniform scenario and

\footnotetext{
${ }^{1}$ The DCI informs the UE about the MCS to be used in the uplink, which is equivalent to $S_{i}^{k}\left(\gamma_{i}^{k}\right)$

${ }^{2}$ Beamforming techniques allow to determine the AoA of a UE.
} 


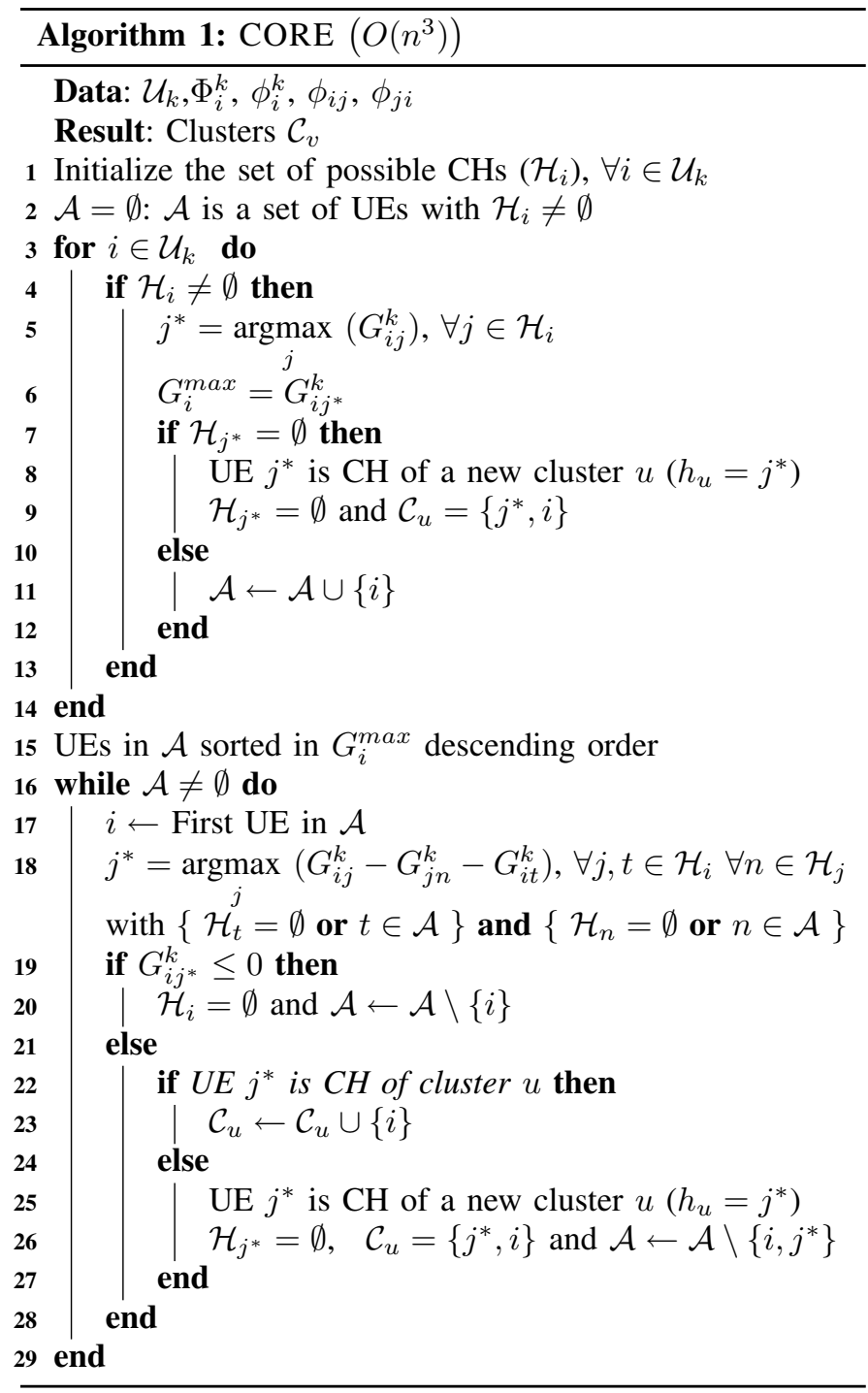

TABLE II: Simulation Parameters

\begin{tabular}{|c|c|}
\hline Bandwidth & $20 \mathrm{MHz}$ \\
\hline Macro cell Path-Loss & $128.1+37.6 \log _{10}(d \mathrm{~km})$ \\
\hline D2D Path-Loss & $148+40 \log _{10}(d \mathrm{~km})$ \\
\hline Macro cell transmitted power & $46 \mathrm{dBm}$ \\
\hline UE transmitted power & $20 \mathrm{dBm}$ \\
\hline D2D transmitted power & $15 \mathrm{dBm}$ \\
\hline
\end{tabular}

the Non-Uniform scenario. In the former, UEs are uniformly spread over the coverage area of the eNB. In the latter, 1/5 of the UEs are uniformly distributed over the coverage area and $4 / 5$ of the UEs are concentrated inside 4 circular hotspots with radius $50 \mathrm{~m}$, whose center is located at the cell edge, 200 $\mathrm{m}$ from the eNB. The total number of UEs ranges from 5 to 55. Regarding the traffic, in both scenarios half of the UEs generate symmetric traffic (i.e VoIP with $64 \mathrm{kbps}$ both in DL and UL), while the rest generate asymmetric traffic (i.e FTP or streaming with 400kbps in the DL and no UL traffic). The rest of the simulations' parameters with their corresponding values, can be found in Table II [15]. In the figures to follow we evaluate the Optimal Clustering (CL) stated in (14), the proposed algorithm (CORE) described in Algorithm 1, and the regular performance without clustering (no $\mathrm{CL}$ ), for both aforementioned scenarios.

Fig. 2a shows the utilization of DL resources in both scenarios. As it may be observed, clustering of UEs can significantly decrease the utilization of DL resources as compared with non clustering. For instance, in both scenarios, when clustering is applied less than $40 \%$ of the available DL spectrum is utilized for 55 UEs. On the contrary, without clustering, the DL resources utilization is $100 \%$ for 50 UEs in the Uniform scenario and for $40 \mathrm{UEs}$ in the Non-Uniform scenario. It may be observed in Fig. 2a that the Uniform scenario always requires less resources than the Non-Uniform scenario (with and without clustering). This is due to the smaller average distance between UEs and serving eNB in the Uniform scenario, and the subsequent better average channel condition.

Unlike DL spectrum utilization, the clustering increases the UL spectrum utilization (see Fig. 2b). This was expected since UL resources are utilized now for both DL and UL traffic within the clusters. However, this trend is counteracted by the sharp decrease in the DL spectrum utilization. For instance, if we consider the Non-Uniform scenario and 40 UEs, it can be seen that clustering reduces the DL utilization by almost $70 \%$ while UL utilization rises $35 \%$. In total, the utilization of resources considering both links is halved. Therefore, efficiency in the usage of resources and balancing of both links (DL and UL) is achieved. It should be also noted that CORE achieves a near optimal DL utilization (Fig. 2a). Yet, the CORE UL utilization is higher than the optimal utilization provided by CL, since CORE reduces the problem complexity at the expense of discarding unlikely cluster configurations. Despite this UL utilization increase, CORE achieves high total clustering gains. Besides, the DL is usually limiting the system's capacity and an excessive UL increase may be limited by the eNB by reducing the number of created clusters.

Fig. $3 \mathrm{a}$ and $3 \mathrm{~b}$ provide us insight on the cluster creation for the two scenarios. Specifically, it can be seen that clusters are created more frequently in the Uniform scenario, while in the Non-Uniform the clusters have more members. This is due to the larger variety of link qualities of the Uniform scenario, that increase the probability of creating clusters. On the contrary, in the Non-Uniform scenario, the percentage of UEs with a bad quality link is high (4/5 of the UEs are located in the cell edge hotspots) but the number of UEs with good quality channel with both the eNB and the possible CMs is reduced. Hence, the probability of creating a cluster is lower and the size of the cluster is bigger.

This can be seen also from Fig. 4 that illustrates the cumulative distribution of the distance between $\mathrm{CHs}$ and eNBs for both scenarios when 55 UEs are deployed. It is interesting to outline that in the Non-Uniform scenario, where only $20 \%$ of the UEs are outside the hotspots, almost $50 \%$ of the $\mathrm{CHs}$ are located less than $150 \mathrm{~m}$ away from the eNB (i.e. clearly outside the hotspots). Therefore, UEs in the hotspots are prone to create clusters with a $\mathrm{CH}$ outside the hotspot. 


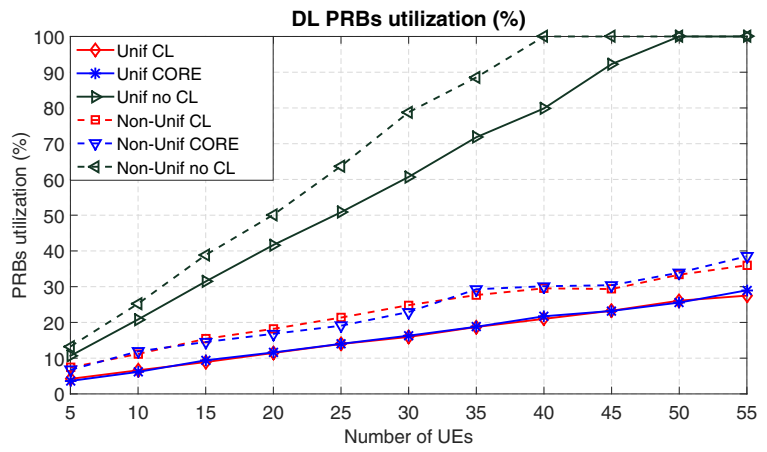

(a) DL spectrum utilization

UL PRBs utilization (\%)

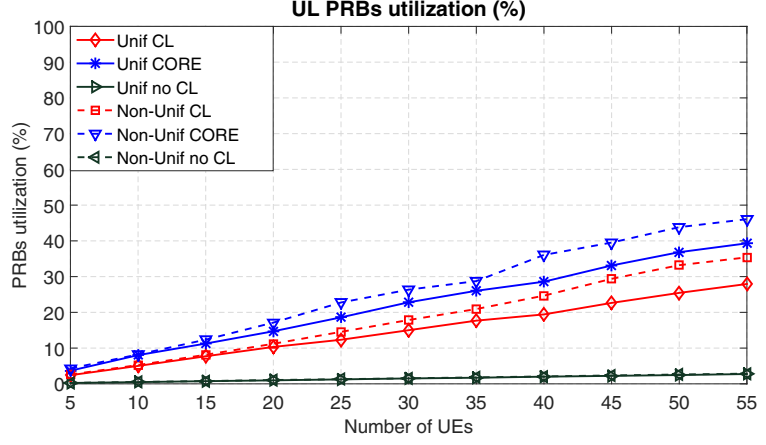

(b) UL spectrum utilization

Fig. 2: DL and UL spectrum utilization

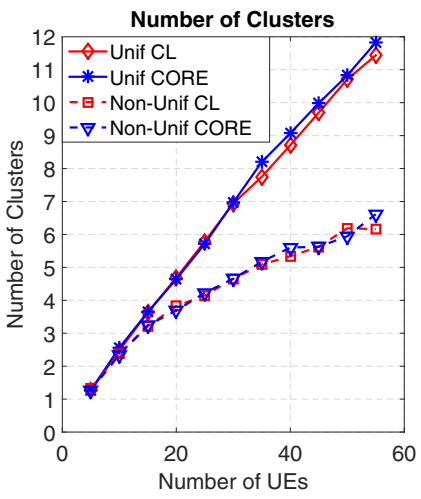

(a) Number of Clusters

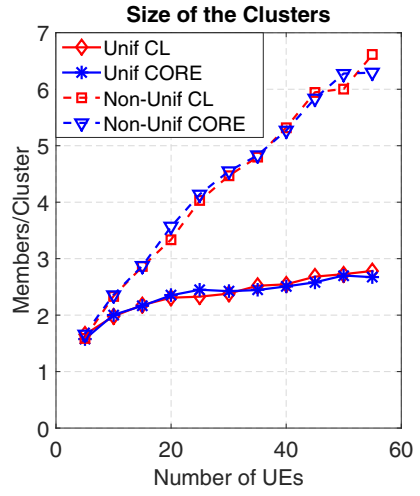

(b) Size of the Clusters
Fig. 3: Number and Size of the Clusters

\section{CONClusions}

In this paper we studied the creation of clusters of UEs to improve the spectral efficiency of the LTE-A network. First, an optimization problem is stated to minimize the utilized resources. Finally, a near-optimal algorithm for clustering is proposed. It has been shown that our proposal can significantly decrease the utilization of resources thus enhancing capacity of cellular networks. Furthermore, the implementation of the proposed clustering algorithm results in a reduction of the UL/DL traffic imbalance.

\section{ACKNOWLEDGEMENT}

This work has been funded by the MITN Project CROSSFIRE (PITN-GA-2012-317126), by the AGAUR (2014SGR

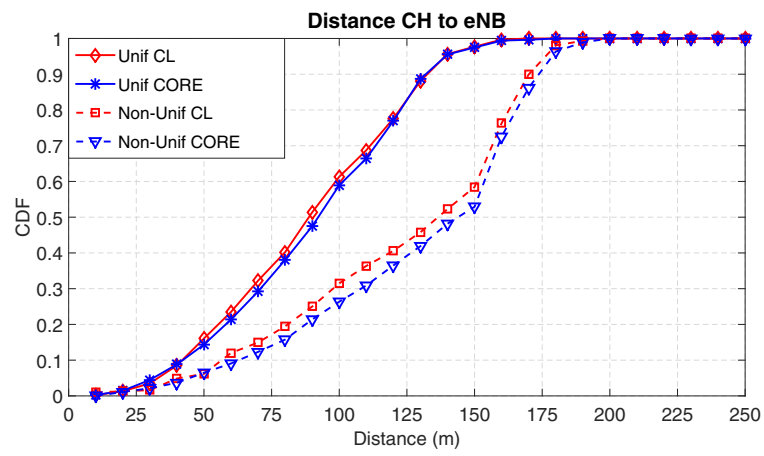

Fig. 4: CH-eNB distance for 55 UEs

1551), by the CellFive (TEC2014-60130-P) and by the Muscles (C2015/1-5) Research Projects.

\section{REFERENCES}

[1] Cisco Visual Networking Index: Global Mobile Data Traffic Forecast Update, 2014-2019, Cisco Systems, Inc, Feb 2015

[2] Damnjanovic, A.; Montojo, J.; Yongbin Wei; Tingfang Ji; Tao Luo; Vajapeyam, M.; Taesang Yoo; Osok Song; Malladi, D., "A survey on $3 G P P$ heterogeneous networks", Wireless Communications, IEEE vol.18, no.3, pp.10,21, June 2011.

[3] Fangming Liu; Peng Shu; Hai Jin; Linjie Ding; Jie Yu; Di Niu; Bo Li, Gearing resource-poor mobile devices with powerful clouds: architectures, challenges, and applications, Wireless Communications, IEEE, vol.20, no.3, pp.14,22, June 2013

[4] 3GPP Technical Report 36.843, Study on LTE device to device proximity services; Radio aspects, V12.0.1, Mar. 2014 Available at www.3gpp.org

[5] Bin Zhou; Honglin Hu; Sheng-Qiang Huang; Hsiao-Hwa Chen, Intracluster Device-to-Device Relay Algorithm With Optimal Resource Utilization, Vehicular Technology, IEEE Transactions on , vol.62, no.5, pp.2315,2326, Jun 2013

[6] Condoluci, M.; Militano, L.; Araniti, G.; Molinaro, A.; Iera, A., Multicasting in LTE-A networks enhanced by device-to-device communications, Globecom Workshops (GC Wkshps), 2013 IEEE , vol., no., pp.567,572, 9-13 Dec. 2013

[7] Seppala, J.; Koskela, T.; Tao Chen; Hakola, S., Network controlled Device-to-Device $(D 2 D)$ and cluster multicast concept for LTE and LTE-A networks, Wireless Communications and Networking Conference (WCNC), 2011 IEEE , vol., no., pp.986,991, 28-31 March 2011

[8] Wang Zhibo; Tian Hui; Chen Nannan, Clustering and power control for reliability improvement in Device-to-Device networks, Globecom Workshops (GC Wkshps), 2013 IEEE , vol., no., pp.573,578, 9-13 Dec. 2013

[9] Daquan Feng; Lu Lu; Yi Yuan-Wu; Li, G.Y.; Gang Feng; Shaoqian $\mathrm{Li}$, Device-to-Device Communications Underlaying Cellular Networks, Communications, IEEE Transactions on , vol.61, no.8, pp.3541,3551, August 2013

[10] White Paper, What is going on in Mobile Broadband Networks? Smartphone Traffic Analysis and Solutions, Nokia Networks, 2014

[11] Wenchi Cheng; Xi Zhang; Hailin Zhang, "Optimal power allocation for full-duplex D2D communications over wireless cellular networks," Global Communications Conference (GLOBECOM), 2014 IEEE , vol., no., pp.4764,4769, 8-12 Dec. 2014

[12] 3GPP Technical Specification 36.213, Physical layer procedures, V12.5.0, Mar. 2015 Available at www.3gpp.org

[13] 3GPP Technical Report 36.942, Radio Frequency (RF) system scenarios, V12.0.0, Oct. 2014 Available at www.3gpp.org

[14] Huan Tang; Zhi Ding; Levy, B.C., "Enabling D2D Communications Through Neighbor Discovery in LTE Cellular Networks," Signal Processing, IEEE Transactions on , vol.62, no.19, pp.5157,5170, Oct.1, 2014

[15] Ali, S.; Rajatheva, N.; Latva-aho, M., "Full duplex device-to-device communication in cellular networks," Networks and Communications (EuCNC), 2014 European Conference on , vol., no., pp.1,5, 23-26 June 2014 